\title{
INDICADORES DE IMPACTO PARA MONITORAR O USO PÚBLICO NO PARQUE ESTADUAL PICO DO MARUMBI - PARANÁ ${ }^{1}$
}

\author{
Leide Yassuco Takahashi², Miguel Serediuk Milano ${ }^{3}$ e Cássio Antonio Tormena ${ }^{4}$
}

\begin{abstract}
RESUMO - Os objetivos deste trabalho foram indentificar e avaliar alguns indicadores e impactos causados pelos visitantes ao Parque Estadual Pico do Marumbi - Paraná, selecionando-se os mais representativos para monitoramento. Os dados foram coletados entre dezembro de 1996 e março de 1997 e os resultados, comparados com áreas sem uso recreativo. Efetuaram-se análise de matriz de correlação, análise fatorial e análise discriminante. Os melhores indicadores para o Parque Marumbi foram: macroporosidade, microporosidade, resistência do solo à penetração de 0-0,05 m e 0,05-0,10 m e densidade do solo.
\end{abstract}

Palavras-chave: Unidades de conservação, recreação e indicadores de impacto.

\section{IMPACT INDICATORS FOR MONITORING RECREATIONAL USE IN THE PICO DO MARUMBI STATE PARK - PARANÁ (BRAZIL)}

\begin{abstract}
The aim of the present study was to analyze some indicators of impact caused by visitors to Pico Marumbi State Park - State of Parana, Brazil, selecting the most significant ones to be monitored. Data were collected from December 1996 to March 1997 and compared with those obtained in adjacent areas, but without recreational use. The results were analyzed using correlation matrix, factorial and discrimant analysis. The most significant indicators for Pico do Marumbi were micro and macroporosity, penetration resistance at $0-5 \mathrm{~cm}$ and $5-10 \mathrm{~cm}$ and bulk density.
\end{abstract}

Key words: Protected areas, recreation and impact indicators.

\section{INTRODUÇÃO}

Com as mudanças econômicas no pós-guerra e o desenvolvimento tecnológico em vários setores, observou-se maior tempo de lazer, resultando num aumento significativo da visitação em áreas naturais. Com isso, a recreação em unidades de conservação vem sendo apresentada como uma alternativa potencial para o desenvolvimento econômico da região onde elas estão inseridas. Entretanto, a escassez de informações, de recursos humanos e financeiros para o planejamento e manejo dessas unidades dificulta a previsão dos impactos da visitação pública aos meios físico e biótico.

A preocupação com os impactos provocados pela recreação em áreas naturais teve início na década de 1930, com avaliações dos efeitos do turismo sobre a vegetação e o solo. De acordo com Lutz (1945) e Cole (1988), o pisoteio dos visitantes compacta o solo e reduz a regeneração natural das espécies vegetais. No final da década de 1950, iniciou-se a utilização do

\footnotetext{
${ }^{1}$ Recebido em 20.01.2004 e aceito para publicação em 25.11.2004.

${ }^{2}$ Professora da Universidade Estadual de Maringá - UEM. E-mail: <ladi@ uem.br>.

${ }^{3}$ Professor da Universidade Federal do Paraná - UFPR. E-mail: <mmilano@ boticário.org.br>.

${ }^{4}$ Professor da Universidade Estadual de Maringá - UEM. E-mail: <catormena@ uem.br>.
} 
conceito de capacidade de carga visando avaliar a demanda e os impactos do uso recreativo (Mc COOL, 1996). Esse modelo foi amplamente utilizado até a década de 1960, quando várias pesquisas comprovaram que não havia uma relação direta entre o número de visitantes e a quantidade de impactos negativos (HAMMITT e COLE, 1998; FENNELL, 2002). Segundo pesquisadores como Mc Cool (1996), Leung e Marion (2000), Magro (1999), Hendee e Dawson (2002) e Drumm e Moore (2002), o paradigma da capacidade de carga fracassou porque ele se preocupava demasiadamente com o número máximo de pessoas, enquanto vários estudos apontavam que muitos problemas do uso recreativo ocorriam em função do comportamento das pessoas (PRISKIN, 2003) e não exatamente do número delas.

Conhecendo as limitações desse modelo de capacidade de carga, técnicos do Serviço Florestal Americano desenvolveram um sistema denominado Limits of Acceptable Changes - LAC (STANKEY et al., 1985). Esse sistema de planejamento pode ser resumido em quatro componentes principais: 1) a definição de indicadores de impactos ecológicos e recreativos; 2) o estabelecimento dos limites máximos aceitáveis de impacto; 3 ) a identificação de ações de manejo necessárias para alcançar essas condições; e 4) um programa de monitoramento e avaliação da efetividade das ações de manejo.

Segundo Merigliano (1987), o termo indicador referese a uma variável específica que, individualmente ou em combinação, é tomada como referência. Além disso, os indicadores podem sinalizar a necessidade de ações corretivas de manejo, avaliar a eficiência e ajudar a determinar se os objetivos da unidade estão sendo atingidos ou não. O termo limite define quanto é aceitável de impacto (STANKEY et al., 1985).

No Brasil, a limitação de recursos, de equipamentos e, principalmente, pessoal é uma constante. Além disso, a maioria dos administradores das unidades de conservação não tem acesso à literatura estrangeira, disponível apenas em algumas bibliotecas especializadas (TAKAHASHI, 1998). Tendo em vista tais aspectos e considerando que o desenvolvimento desordenado da recreação em unidades de conservação brasileiras pode comprometer os objetivos para os quais elas foram estabelecidas (MILANO, 2000), é fundamental realizar uma investigação sistemática sobre os impactos do uso recreativo para descobrir novos fatos ou princípios.
Nesse contexto, os objetivos deste estudo foram avaliar os principais indicadores ecológicos de impacto da área de acampamento do Parque Estadual Pico do Marumbi, selecionando-se os indicadores que melhor representavam os efeitos dos impactos nessa área.

\section{MATERIAL E MÉTODOS}

O presente estudo foi conduzido nas áreas de acampamento selvagem (pequenas clareiras dentro da zona de uso extensivo) do Parque Estadual Pico do Marumbi, situado no domínio da Mata Atlântica, mais especificamente na Floresta Ombrófila Densa (IBGE, 1992). O parque possui uma área de 2.342 ha, localizase no município de Morretes, a $65 \mathrm{~km}$ de Curitiba, e, desde sua abertura ao público, em junho de 1995, até dezembro de 1997, recebeu cerca de 20.400 visitantes. Por ser uma área tradicionalmente utilizada para caminhadas e escaladas, o maior fluxo de visitantes ocorre nos meses mais secos (entre maio e agosto). A infra-estrutura básica do parque compreende o centro de visitantes, centro administrativo, museu, posto da polícia florestal, laboratório, alojamento para pesquisadores e estagiários e sede para equipe de resgate.

As sete clareiras destinadas a acampamentos localizam-se em áreas de Floresta Ombrófila Densa Montana ou de transição entre Submontana e Montana, cujo solo resulta da Associação de Cambissolo Podzóilco álico $\mathrm{Tb}$ textura média/argilosa relevo forte ondulado/ montanhoso substrato granito + solos Litólicos álicos Tb textura siltosa/média relevo montanhoso, ambos A húmico fase floresta ombrófila densa submontana e montana. São pequenos locais com uma área média de $33 \mathrm{~m}^{2}$, desmatados para fins de acampamento.

Com base na metodologia proposta por Settergren e Cole (1970) e Cole (1989), estabeleceram-se três áreastestemunha para posterior comparação entre os resultados obtidos nas áreas de uso público e as áreas fechadas à visitação.

Fundamentados em estudos desenvolvidos por Cole (1982, 1983, 1987 e 1989), Marion e Merriam (1985) e Stohlgren e Parsons (1986), foram selecionados os seguintes indicadores:

a) Regeneração natural no entorno da clareira Adaptando a proposta de Cole (1982) e Cole e Hall (1992) para quantificar a regeneração arbórea, estabeleceram-se oito áreas amostrais, aleatoriamente 
sorteadas. Cada área amostral totalizava $1 \mathrm{~m}^{2}$, e foram registradas todas as plântulas com altura superior a $15 \mathrm{~cm}$ e DAP máximo de $5 \mathrm{~cm}$.

b) Resistência do solo à penetração - Como relataram Cole (1989) e Cole e Hall (1992), a resistência à penetração é uma medida sensível e de fácil obtenção. Neste estudo, ela foi determinada utilizando-se um penetrômetro estático modelo Solotest (haste metálica e cone com área basal de $1 \mathrm{~cm}^{2}$ e semi-ângulo de $30^{\circ}$ ), conforme metodologia descrita por Tormena e Roloff (1996). Em cada clareira foram amostrados oito pontos sorteados aleatoriamente. Em cada ponto foram realizadas medidas nas profundidades de $0-5 \mathrm{~cm}$ e de $5-10 \mathrm{~cm}$, três dias após uma chuva suficiente para umedecer o perfil amostrado.

c) Densidade do solo - Realizado conforme as sugestões de Lutz (1945), Cole (1982) e Cole (1989). Foram obtidas amostras com estrutura não-deformada, coletadas utilizando-se um amostrador com anéis volumétricos de $50 \mathrm{~cm}^{3}$, próximos ao ponto onde foram realizadas as leituras de resistência à penetração. A densidade do solo foi determinada conforme Blake e Hartge (1986).

d) Microporosidade - Determinada como o volume de água retida nas amostras após sua drenagem no potencial de $-0,006 \mathrm{MPa}$, obtida nas amostras com estrutura não-defromada utilizadas para a determinação da densidade do solo.

e) Macroporosidade - Obtida por meio do volume de água drenada com a aplicação do potencial de 0,006 MPa, após saturação das amostras com estrutura não-deformada utilizadas para a determinação da densidade do solo.

f) Conteúdo de carbono orgânico do solo - Obtido em amostras deformadas coletadas em cada uma das clareiras, próximas aos pontos de coleta das outras variáveis. O teor de carbono orgânico do solo foi determinado utilizando-se a metodologia descrita pela Embrapa (1997).

g) Máxima capacidade de retenção de água ou umidade na capacidade de campo - Obtida nas amostras indeformadas como o volume de água retida após a aplicação do potencial equivalente a -0,01 MPa, conforme Reichardt (1988). A determinação da capacidade de campo foi efetuada em mesa de tensão (EMBRAPA, 1997).

h) Granulometria do solo-A distribuição do tamanho de partículas sólidas do solo foi determinada em amostras deformadas coletadas em cada uma das clareiras, próximo aos pontos de coleta das outras variáveis. Utilizouse o método da pipeta descrito pela Embrapa (1997).

As amostras das diferentes variáveis avaliadas foram obtidas entre dezembro/96 e março/97 e as análises, conduzidas no Laboratório de Física e Química de Solos do Departamento de Agronomia da Universidade Estadual de Maringá, PR.

Para a análise estatística das variáveis indicadoras, obteve-se inicialmente a matriz de correlação para avaliar o grau de associação entre elas. Em seguida, efetuouse a análise fatorial pelo método das componentes principais, utilizando-se a rotação varimax normalizada, a fim de reduzir o conjunto de variáveis, agrupando em fatores as variáveis correlacionadas, o que permitiu identificar a estrutura dos indicadores de impacto selecionados. Após essa avaliação, realizou-se uma análise discriminante (stepwise analysis) para selecionar os indicadores que diferenciavam melhor as áreastestemunha das áreas de uso recreativo. Por fim, efetuouse a padronização das variáveis em torno da média e do desvio-padrão, para corrigir as diferenças de escala das variáveis.

Os resultados das análises foram obtidos através do software Statistica, utilizando-se os módulos Discriminant analysis e Factorial analysis.

\section{RESULTADOS E DISCUSSÃO}

Os resultados das análises de amostras de solo e regeneração natural são apresentados no Anexo 1 .

De acordo com a matriz de correlação apresentada na Tabela 1, observa-se que as correlações mais altas são encontradas na resistência à penetração nas camadas de 0-0,05 m e 0,05-0,10 m de profundidade $(\mathrm{r}=0,85)$ e também entre os indicadores capacidade de retenção de água e microporosidade $(\mathrm{r}=0,96)$.

A regeneração natural indica correlações mais elevadas com o conteúdo de carbono e densidade do solo. Os resultados apontaram que a regeneração natural de plantas é positivamente correlacionada com as variáveis conteúdo de carbono orgânico, macroporosidade, microporosidade e capacidade de campo e negativamente com a densidade do solo. Isso indica que a regeneração, ou seja, o número de plantas por $\mathrm{m}^{2}$ é dependente da qualidade estrutural do solo, refletida pela porosidade do solo, a qual é dependente dos níveis de matéria orgânica do solo.

R. Árvore, Viçosa-MG, v.29, n.1, p.159-167, 2005 
Tabela 1 - Correlação entre os indicadores ecológicos de impacto do uso recreativo, encontrados no "camping" selvagem do Parque Estadual Pico do Marumbi. Morretes, PR, 1996.

Table 1 - Correlation of different ecological indicators of recreational impact at camping area in Pico Marumbi State Park. Morretes, PR, 1996.

\begin{tabular}{|c|c|c|c|c|c|c|c|c|}
\hline Indicadores & $\mathrm{RP}(5)$ & $\mathrm{RP}(10)$ & $\mathrm{C}$ & Reg. & Dens. & Mac. & Mic. & C.R.ag \\
\hline RP (5) - Resistência à penetração - 0-5 cm (MPa) & - & & & & & & & \\
\hline $\mathrm{RP}(10)$ - Resistência à penetração - 5-10 cm (MPa) & 0.85 & - & & & & & & \\
\hline $\mathrm{C}$ - Conteúdo de carbono $\left(\mathrm{g} \mathrm{dm}^{-3}\right)$ & 0.22 & 0.36 & - & & & & & \\
\hline Reg. -Regeneração natural $\left(\mathrm{n}^{\circ}\right.$ plantas $\left./ \mathrm{m}^{2}\right)$ & 0.09 & 0.13 & 0.48 & - & & & & \\
\hline Dens. - Densidade do solo $\left(\mathrm{g} \mathrm{dm}^{-3}\right)$ & 0.25 & 0.17 & -0.58 & -0.53 & - & & & \\
\hline Mac. - Macroporosidade $\left(\mathrm{m}^{3} \mathrm{~m}^{-3}\right)$ & -0.18 & -0.17 & -0.39 & 0.28 & -0.54 & - & & \\
\hline Mic. - Microporosidade $\left(\mathrm{m}^{3} \mathrm{~m}^{-3}\right)$ & -0.12 & -0.11 & 0.19 & 0.37 & -0.59 & -0.24 & - & \\
\hline C.R.ag. - Capacidade de retenção de água $\left(\mathrm{m}^{3} \mathrm{~m}^{-3}\right)$ & -0.11 & -0.12 & 0.14 & 0.27 & -0.51 & -0.30 & 0.96 & - \\
\hline
\end{tabular}

A macroporosidade, a microporosidade e a capacidade de campo são negativamente correlacionadas com a densidade do solo, evidenciando-se que um aumento na densidade do solo tem impactos negativas na difusão de oxigênio no solo, bem como na disponibilidade de água às plantas. Além disso, as correlações positivas entre densidade e resistência à penetração indicaram maior sensibilidade do solo em aumentar a resistência com o seu secamento, o que foi corroborado por Tormena et al. (1998). Em concordância com Tormena e Roloff (1996), a resistência à penetração, nas duas profundidades, é positivamente relacionada com a densidade e, negativamente, com a retenção de água. Esse fato indica que o impacto do pisoteio aumenta a densidade do solo, o que pode resultar em aumento também da resistência à penetração, devido à forte dependência da resistência em relação à umidade do solo.

Os resultados da análise fatorial estão apresentados na Tabela 2. A estrutura dimensional dessa análise compreende três fatores, os quais explicam cerca de $80 \%$ da variância total. Observando os resultados da análise fatorial (Tabela 2), constatou-se que o fator 1 possui, como variáveis de maior peso, os indicadores conteúdo de carbono, macroporosidade, densidade do solo e regeneração natural, explicando $30 \%$ da variância total, em que o conteúdo de carbono, a regeneração natural e a macroporosidade são negativamente relacionados com a densidade do solo. Tal situação ratifica a afirmação de diversos autores, entre os quais Kiehl (1979), de que quanto mais elevada for a densidade do solo, maiores serão as limitações para o crescimento e desenvolvimento das plantas.

Tabela 2 - Análise fatorial dos indicadores ecológicos de impacto do uso recreativo no "camping" selvagem do Parque Estadual Pico do Marumbi, pelo método das componentes principais. Morretes, PR, 1996

Table 2 - Factorial analysis of ecological indicators of recreational impact at camping area in Pico Marumbi State Park. Morretes, $P R, 1996$

\begin{tabular}{lccc}
\hline & PESO DAS VARIÁVEIS NOS FATORES & \\
\hline Variável & Fator 1 & Fator 2 & Fator 3 \\
\hline Resistência à penetração de 0-5 cm (MPa) & -0.006406 & 0.937120 & 0.062915 \\
Resistência à penetração de 5-10 cm (MPa) & 0.081897 & 0.953613 & 0.06521 \\
Conteúdo de carbono $\left(\mathrm{g} \mathrm{dm}^{-3}\right)$ & 0.805384 & 0.329327 & -0.076070 \\
Regeneração natural $\left(\mathrm{n}^{0} \mathrm{plantas}^{-} \mathrm{m}^{2}\right)$ & 0.693177 & 0.146634 & -0.283104 \\
Densidade do solo $\left(\mathrm{g} \mathrm{dm}^{-3}\right)$ & -0.821914 & 0.261622 & 0.408380 \\
Macroporosidade $\left(\mathrm{m}^{3} \mathrm{~m}^{-3}\right)$ & 0.762331 & -0.294204 & 0.469184 \\
Microporosidade $\left(\mathrm{m}^{3} \mathrm{~m}^{-3}\right)$ & 0.202447 & -0.076966 & -0.962793 \\
Capacidade de retenção de água $\left(\mathrm{m}^{3} \mathrm{~m}^{3}\right)$ & 0.108358 & -0.074269 & -0.968098 \\
Explicação da variância & 2.445302 & 2.083970 & 2.344769 \\
Proporção total & 0.305663 & 0.260496 & 0.293096 \\
\hline
\end{tabular}

R. Árvore, Viçosa-MG, v.29, n.1, p.159-167, 2005 
O fator 2 é determinado pelo indicador resistência à penetração, em dois níveis de profundidade, e explica $26 \%$ da variância total. O fator 3 , cujas variáveis de maior peso na análise são os indicadores capacidade de campo e a microporosidade explica $29 \%$ da variância total. Esse resultado pode ser justificado pelo fato de a retenção de água no solo ocorrer nos microporos e mesoporos do solo (KIEHL, 1979), os quais estão contidos nas duas variáveis indicadoras. Aumento na densidade do solo devido ao intenso pisoteio pelos visitantes tem como conseqüência, simultaneamente, o aumento da resistência à penetração e redução na capacidade de retenção de água pelo solo, o que justifica tais resultados. Todos esses indicadores estão ligados aos efeitos negativos que o pisoteio dos visitantes e a perda da vegetação exercem sobre o solo.

Os resultados da análise discriminante (Tabela 3) indicam que a macroporosidade é o indicador mais importante para a área e, na seqüência, o que melhor discrimina as áreas de acampamento das áreas sem uso recreativo é a microporosidade. De acordo com Cole (1985), os efeitos da compactação do solo em área de acampamento têm sido constatados freqüentemente e resultam em mudanças na porosidade, por exemplo a perda de até $60 \%$ de macroporos em determinadas áreas. A macroporosidade é facilmente modificada pela aplicação de carga ao solo (pisoteio), o que tem sido comprovado em inúmeros estudos relacionados com os efeitos do manejo e da compactação em solos agrícolas.

Tabela 3 - Variáveis de impacto ecológico que melhor discriminam as clareiras de acampamento das áreas sem uso recreativo no "camping" selvagem do Parque Estadual Pico do Marumbi, Morretes, PR, 1996

Table 3 - Best ecological impact indicators to discriminate between camping area and non-recreative areas at Pico Marumbi State Park. Morretes, PR, 1996

\begin{tabular}{lcc}
\hline Variáveis & F-remove $(1,75)$ & p-level \\
\hline Macroporosidade $\left(\mathrm{m}^{3} \mathrm{~m}^{-3}\right)$ & 31.21744 & 0.000000 \\
Microporosidade $\left(\mathrm{m}^{3} \mathrm{~m}^{-3}\right)$ & 7.81904 & 0.006563 \\
Resistência do solo & 2.74781 & 0.101562 \\
à penetração de $5-10 \mathrm{~cm}(\mathrm{MPa})$ & & \\
$\begin{array}{l}\text { Resistência do solo } \\
\text { à penetração de } 0-5 \mathrm{~cm}(\mathrm{MPa})\end{array}$ & 1.24802 & 0.267496 \\
\hline
\end{tabular}

Tendo em vista a alta correlação observada na Tabela 1, entre os indicadores macroporosidade, microporosidade e densidade do solo, o fato de esses indicadores apresentarem pesos elevados nos fatores 1 e 3 (Tabela 2) e, ainda, o fato de a macroporosidade discriminar melhor as duas áreas, pode-se simplificar o processo de seleção dos indicadores, preconizando e utilizando no monitoramento esses três indicadores. $\mathrm{O}$ fato de esses indicadores serem obtidos de uma amostra representando a estrutura natural do solo, além de serem de determinação relativamente fácil, justifica a sugestão de utilizá-los no monitoramento dessa área de estudo. Ademais, são medidas que podem ser temporalmente realizadas, permitindo acompanhar a evolução da qualidade do solo em função da visitação pública nas áreas de recreação.

Considerando que essas variáveis representam o estado físico atual do solo, vale ressaltar que elas variam por efeito da compactação ou pela incorporação de matéria orgânica, o que depende da forma e da intensidade do uso recreativo e do manejo adotado. Isso é bastante positivo ao considerar-se que existem alternativas de manejo simples, como a incorporação de matéria orgânica (galhos e folhas em decomposição que podem ser trazidos das proximidades) e suspensão temporária da visitação, as quais podem ser adotadas para melhorar as propriedades físicas do solo e promover a regeneração das plantas.

De acordo com os resultados das análises de amostras de solo obtidos no Marumbi (Anexo 1), as condições físicas do solo ainda não comprometem a regeneração natural e o crescimento das plantas. No entanto, é essencial o monitoramento da área para observar por quanto tempo essa condição persiste e de que forma algumas alternativas de manejo podem garantir a continuidade dessa condição.

$\mathrm{O}$ fato de os indicadores regeneração natural e densidade do solo discriminarem as áreas de acampamento das áreas sem uso recreativo, juntamente com a macroporosidade e a microporosidade, como indica o resultado da análise discriminante apresentada na Tabela 3, confirma que a compactação, provocada pelo pisoteio, é prejudicial ao solo e à vegetação. Além de problemas ao crescimento das raízes, a compactação dificulta a infiltração de água no solo, resultando em solo exposto, erosão e perdas de solo e, em casos extremos, exposição das raízes das plantas, como atestaram Leung e Marion (1999).

R. Árvore, Viçosa-MG, v.29, n.1, p.159-167, 2005 
No Marumbi, a resistência à penetração média na superfície $(0-0,05 \mathrm{~m})$ foi de $0,78 \mathrm{MPa}$ nas clareiras e $0,69 \mathrm{MPa}$ nas testemunhas, enquanto na profundidade de 0,05-0,10 $\mathrm{m}$ as médias obtidas foram 0,74 MPa nas clareiras e 0,71 MPa nas testemunhas, havendo diferença significativa nos dois casos $(\mathrm{p}<0,05)$. Tormena e Roloff (1996) destacaram que muitos autores adotavam para a resistência à penetração um limite de 1,0 MPa como valor crítico e 2,0 MPa como impeditivo ao crescimento das raízes. Embora a situação observada no Marumbi se aproxime do valor crítico, acredita-se que, antes de adotar qualquer medida, novas avaliações devam ser realizadas, a fim de verificar os níveis de resistência à penetração do solo.

O fato de quatro indicadores ecológicos apresentados na Tabela 3 discriminarem as áreas de acampamento das áreas-testemunha não significa que eles devam ser monitorados imediatamente, mas que conseguem, hoje, estabelecer melhor as diferenças entre as duas áreas. As condições das testemunhas em relação às áreas utilizadas para acampar nada mais são do que um ponto de referência para futuras comparações. Elas não podem ser consideradas condições ideais, uma vez que, embora sem uso, a área não esteja completamente protegida da ação dos visitantes e processos naturais.

Em um primeiro momento, pode-se dizer que a escolha de cada indicador depende inicialmente da representatividade individual de cada um e, depois, da correlação que apresente com os outros. Dessa forma, consideram-se como bons indicadores a serem monitorados no caso da área de "camping" selvagem do Parque Marumbi as variáveis macroporosidade; microporosidade; resistência à penetração nas camadas de 0,05-0,10 m e de 0-0,05 $\mathrm{m}$ de profundidade; e, também, a densidade do solo.

\section{CONCLUSÕES}

A metodologia adotada foi eficiente para identificar os principais indicadores de impacto no solo, e a definição destes permite aos administradores otimizar o uso dos recursos, priorizando o monitoramento daqueles que melhor refletem as condições da área.

O monitoramento das áreas avaliadas com base na seleção dos indicadores mais representativos poderá subsidiar as decisões futuras de manejo. Os principais indicadores ecológicos a serem monitorados no
Parque Marumbi são a macroporosidade, a microporosidade, a resistência do solo à penetração na superfície e entre 0,05 e $0,10 \mathrm{~m}$ e a densidade do solo.

É essencial que as pesquisas continuem e sejam divulgadas. Como o processo de planejamento é dinâmico, poderá haver necessidade de substituir alguns indicadores que reflitam melhor as condições reais de cada área.

\section{REFERÊNCIAS BIBLIOGRÁFICAS}

BLAKE, G.R.; HARTGE, K.H. Bulk density. In: Klute, A. (Ed.) Methods of soil analysis: physical and mineralogical methods. 2. ed. Madison: America Society of Agronomy, 1986. v.13. p.363-375.

COLE, D.N. Wilderness campsite impacts: Effect of amount of use. Research Paper INT, n.284, p.1-34, 1982.

. Monitoring the condition of wilderness campsites. Research Paper INT, n.302, p.110. 1983.

Low-impact recreational practices for wilderness and backcountry. General Technical Report INT, n.265, p.1-131, 1985.

. Recreational impacts on backcountry campsites in Grand Canyon National Park.

Environment Management, n.10, p.651659, 1987.

Disturbance and recovery of trampled montane grassland and forests in Montana. Research Paper INT, n.389, p.1-37, 1988.

Wilderness campsite monitoring methods: A sourcebook. General Technical Report INT, n. 259, p. 1-57, 1989.

COLE, D.N.; HALL, T.E. Trends in campsite condition: Eagle Cap Wilderness, Bob Marshall Wilderness, and Grand Canyon National Park. Research Paper INT, n.453, p.1-40, 1992.

DRUMM, A.; MOORE, A. Desenvolvimento do Ecoturismo - um manual para profissionais de conservação. Virgínia: The Nature Conservancy, 2003. v. 1. 100p. 
EMPRESA BRASILEIRA DE PESQUISA AGROPECUÁRIA - EMBRAPA. Serviço Nacional de Levantamento e Conservação de Solos. Manual de Métodos de Análises de Solos. Rio de Janeiro: 1997.

FENNELL, D.A. Ecoturismo - uma introdução. São Paulo: Contexto, 2002. 281p.

FUNDAÇÃO INSTITUTO BRASILEIRO DE GEOGRAFIA E ESTATÍSTICA (FIBGE). Manual técnico da vegetação brasileira. Rio de Janeiro: 1992. (Manuais Técnicos de Geociências, n. 1).

HAMMITT, W.E.; COLE, D.N. Wildland recreation - ecology and management. 2. ed. New York: John Wiley \& Sons, 1998. 361p.

HENDEE, J.C.; DAWSON, C.P. Wilderness Management - stewardships and protection of resources and values. 3 . ed. Ogden: Fulcrum Publishing, 2002. p.637.

KIEHL, E.J. Manual de edafologia - relação solo-planta. São Paulo: CERES, 1979. p.262.

LEUNG, Y:; MARION, J.L. Characterizing backcountry camping impacts in Great Smoky Mountains National Park. Journal of Environmental Management, v.57, p.193-203, 1999.

LEUNG, Y.; MARION, J.L. Recreation Impacts and Management in wilderness: a state-of knowledge review. USDA Forest Service proceedings RMRS. v. 5, p.15. 2000.

LUTZ, H.J. Soil condition of picnic grounds in public forest parks. Journal of Forestry, n.43, p.121-127, 1945.

MAGRO, T.C. Impactos do uso público em uma trilha do parque Nacional do Itatiaia. 1999. 135f. Tese (Doutorado) Universidade de São Paulo. 1999.

MARION, J.L.; MERRIAN, L.C. Predicability of recreational impact on soils. Soil Science Society of America Journal, v.49, n.3, p.751-753, 1985.

Mc COOL, S.F. Limits of acceptable change: A framework for managing national protected areas: experiences from the United States. In: WORKSHOP ON IMPACT MANAGEMENT IN MARINE PARKS 1996, Kuala Lumpur. Paper... Maritime Institute of Malaysia, Kuala Lumpur: 1996. (Endereço eletrônico).
MERIGLIANO, L. The identification and evaluation of indicators to monitor wilderness conditions. 1987. $273 \mathrm{f}$. Dissertação (Master of Science) University of Idaho - Moscow, 1987.

MILANO, M.S. Mitos no manejo de unidades de conservação no Brasil, ou a verdadeira ameaça. In: CONGRESSO BRASILEIRO DE UNIDADES DE CONSERVAÇÃO, 2., 2000, Campo Grande. Anais... Curitiba: Rede Pró-Unidades de Conservação, 2000. p.11-25.

PRISKIN, J. Tourist perceptions of degradation caused by coastal nature-based recreation. Environmental Management, v.32, n.2, p.189-204, 2003.

REICHARDT, K. Capacidade de campo. Revista Brasileira de Ciência do Solo, v.12, p.211-216, 1988.

SETTERGREN, C.D.; COLE, D.M. Recreation effects on soil and vegetation in the Missouri Ozarks. Journal of Forestry, v.68, n.4, p.231233,1970 .

STANKEY, G.H. et al. The Limits of Acceptable Change (LAC) system for wilderness planning. General Technical Report INT, n. 176, p.1-37, 1985.

STOHLGREN, T.J.; PARSONS, D.J. Vegetation and soil recovery in wilderness campsites closed to visitor use. Environmental Management, n.10, p.375-380, 1986.

TAKAHASHI, L. Y. Caracterização dos visitantes, suas preferências e percepções e avaliação dos impactos da visitação pública em duas unidades de conservação do Estado do Paraná. 1998. 129f. Tese (Doutorado) - Universidade Federal do Paraná, Curitiba, 1998.

TORMENA, C.A.; SILVA, A.P.; LIBARDI, P.L. Caracterização do intervalo hídrico ótimo de um latossolo roxo sob plantio direto. Revista Brasileira de Ciência do Solo, v.22, p.573-581, 1998.

TORMENA, C.A.; ROLOFF, G. Dinâmica da resistência à penetração de um solo sob plantio direto. Revista Brasileira de Ciência do Solo, n.20, p.333-339, 1996.

R. Árvore, Viçosa-MG, v.29, n.1, p.159-167, 2005 
ANEXO 1 - INDICADORES DE IMPACTO DO PARQUE MARUMBI

\begin{tabular}{|c|c|c|c|c|c|c|c|c|c|c|c|c|}
\hline Variáv & $\begin{array}{c}\text { el } \mathrm{V} 1 \\
\mathrm{RP}(0-5) \\
\mathrm{MPa}\end{array}$ & $\begin{array}{c}\mathrm{V} 2 \\
\mathrm{RP}(5-10) \\
\mathrm{MPa}\end{array}$ & $\begin{array}{c}\mathrm{V} 3 \\
\mathrm{C} \\
\mathrm{g} \mathrm{cm}-3\end{array}$ & $\begin{array}{c}\text { V4 } \\
\text { Reg. } \\
\text { n.pl/m2 }\end{array}$ & $\begin{array}{c}\text { V5 } \\
D_{\mathrm{s}} \\
\mathrm{gdm}-3 \\
\end{array}$ & $\begin{array}{c}\mathrm{V6} \\
\mathrm{Mac} \\
\mathrm{m} 3 \mathrm{~m}-3\end{array}$ & $\begin{array}{c}\mathrm{V7} \\
\mathrm{Mic} \\
\mathrm{m} 3 \mathrm{~m}-3\end{array}$ & $\begin{array}{c}\mathrm{V} 8 \\
\mathrm{CRA} \\
\mathrm{m} 3 \mathrm{~m}-3\end{array}$ & $\begin{array}{c}\text { AG } \\
\%\end{array}$ & $\begin{array}{r}\mathrm{AF} \\
\% \\
\end{array}$ & $\begin{array}{c}\text { Sil. } \\
\%\end{array}$ & $\begin{array}{c}\operatorname{Arg} \\
\%\end{array}$ \\
\hline 1 & 1,2263 & 1,14205 & 32,3 & 9 & 1,1232 & 0,0096 & 0,528 & 0,5021 & 35 & 10 & 27 & 28 \\
\hline 1 & 1,0657 & 0,95258 & 30,02 & 1 & 1,1933 & 0,0457 & 0,458 & 0,4534 & 40 & 8 & 26 & 26 \\
\hline 1 & 1,4841 & 1,42624 & 34,58 & 0 & 1,1555 & 0,0073 & 0,507 & 0,4779 & 34 & 10 & 30 & 26 \\
\hline 1 & 0,7473 & 0,78943 & 39,14 & 0 & 0,9048 & 0,0061 & 0,58 & 0,5801 & 31 & 9 & 32 & 28 \\
\hline 1 & 0,9421 & 0,96837 & 33,44 & 8 & 1,0686 & 0,0141 & 0,533 & 0,5258 & 43 & 8 & 23 & 26 \\
\hline 1 & 1,1789 & 1,11047 & 25,46 & 8 & 1,2275 & 0,0128 & 0,497 & 0,4751 & 42 & 8 & 22 & 28 \\
\hline 1 & 1,6894 & 1,12626 & 20,9 & 9 & 1,2631 & 0,0288 & 0,478 & 0,4187 & 31 & 8 & 25 & 36 \\
\hline 1 & 1,2947 & 0,73154 & 19,76 & 0 & 1,1947 & 0,0054 & 0,524 & 0,5168 & 39 & 9 & 21 & 31 \\
\hline 1 & 0,7315 & 0,66312 & 25,08 & 12 & 0,9016 & 0,0046 & 0,647 & 0,6466 & 21 & 13 & 31 & 35 \\
\hline 1 & 0,8736 & 0,71049 & 24,32 & 28 & 0,8538 & 0,013 & 0,644 & 0,6397 & 21 & 10 & 34 & 35 \\
\hline 1 & 0,9526 & 0,99995 & 35,34 & 29 & 0,8217 & 0,01 & 0,64 & 0,6191 & 26 & 10 & 32 & 32 \\
\hline 1 & 0,8842 & 0,921 & 36,86 & 29 & 0,8481 & 0,0808 & 0,574 & 0,5719 & 22 & 11 & 33 & 34 \\
\hline 1 & 0,9421 & 0,98942 & 31,16 & 27 & 0,9604 & 0,0174 & 0,592 & 0,5188 & 24 & 13 & 30 & 33 \\
\hline 1 & 0,6315 & 0,68944 & 34,2 & 15 & 0,8752 & 0,011 & 0,621 & 0,617 & 20 & 9 & 40 & 31 \\
\hline 1 & 0,7315 & 0,83154 & 31,16 & 17 & 0,8741 & 0,0188 & 0,609 & 0,6049 & 24 & 13 & 32 & 31 \\
\hline 1 & 0,8579 & 0,59997 & 19,76 & 20 & 0,9102 & 0,0023 & 0,646 & 0,6461 & 21 & 10 & 33 & 36 \\
\hline 1 & 0,5052 & 0,42629 & 19,38 & 3 & 0,9158 & 0,1068 & 0,488 & 0,487 & 26 & 17 & 23 & 34 \\
\hline 1 & 0,5631 & 0,62102 & 25,46 & 12 & 0,9915 & 0,0497 & 0,505 & 0,4753 & 26 & 15 & 26 & 33 \\
\hline 1 & 0,5473 & 0,58418 & 23,94 & 11 & 0,9437 & 0,0303 & 0,548 & 0,5019 & 26 & 15 & 27 & 32 \\
\hline 1 & 0,6052 & 0,61576 & 20,9 & 7 & 1,0273 & 0,0098 & 0,538 & 0,5082 & 24 & 15 & 26 & 35 \\
\hline 1 & 0,5526 & 0,54208 & 27,74 & 11 & 0,9831 & 0,0027 & 0,58 & 0,5738 & 27 & 18 & 16 & 39 \\
\hline 1 & 0,5842 & 0,54734 & 19,38 & 10 & 1,048 & 0,0015 & 0,564 & 0,5637 & 27 & 18 & 18 & 37 \\
\hline 1 & 0,7052 & 0,62102 & 22,42 & 3 & 1,1301 & 0,0052 & 0,543 & 0,5432 & 28 & 21 & 18 & 33 \\
\hline 1 & 0,521 & 0,61049 & 23,56 & 3 & 0,9826 & 0,0131 & 0,544 & 0,5134 & 26 & 21 & 15 & 38 \\
\hline 1 & 0,6473 & 0,68417 & 20,14 & 15 & 0,8805 & 0,0226 & 0,6158 & 0,6124 & 18 & 14 & 19 & 49 \\
\hline 1 & 0,7473 & 0,71575 & 23,56 & 21 & 0,9933 & 0,0151 & 0,562 & 0,5459 & 21 & 16 & 18 & 45 \\
\hline 1 & 0,6579 & 0,75259 & 38 & 15 & 0,6293 & 0,0159 & 0,691 & 0,6809 & 20 & 12 & 29 & 39 \\
\hline 1 & 1,1631 & 0,81048 & 27,36 & 18 & 1,0154 & 0,0948 & 0,454 & 0,4443 & 24 & 21 & 14 & 41 \\
\hline 1 & 1,0894 & 0,95784 & 22,8 & 53 & 1,1004 & 0,0422 & 0,53 & 0,5137 & 25 & 18 & 19 & 38 \\
\hline 1 & 0,8947 & 0,81575 & 22,8 & 5 & 0,9239 & 0,0314 & 0,571 & 0,5442 & 20 & 18 & 16 & 46 \\
\hline 1 & 0,6947 & 0,6947 & 21,66 & 22 & 0,8738 & 0,0197 & 0,618 & 0,5918 & 18 & 14 & 21 & 47 \\
\hline 1 & 0,6736 & 0,59471 & 23,94 & 19 & 0,8264 & 0,0316 & 0,615 & 0,6064 & 17 & 14 & 19 & 50 \\
\hline 1 & 0,621 & 0,5105 & 21,66 & 6 & 0,8808 & 0,0197 & 0,611 & 0,6093 & 18 & 14 & 24 & 44 \\
\hline 1 & 0,5526 & 0,53155 & 23,94 & 8 & 0,7422 & 0,0294 & 0,646 & 0,631 & 19 & 13 & 25 & 43 \\
\hline 1 & 0,7842 & 0,49997 & 22,04 & 6 & 0,9446 & 0,1377 & 0,556 & 0,5426 & 20 & 14 & 26 & 40 \\
\hline 1 & 0,921 & 0,79996 & 30,02 & 2 & 1,0517 & 0,0416 & 0,547 & 0,5434 & 23 & 18 & 25 & 34 \\
\hline 1 & 0,6473 & 0,77364 & 38,38 & 34 & 0,6671 & 0,0488 & 0,668 & 0,6275 & 16 & 10 & 43 & 31 \\
\hline 1 & 0,6789 & 0,68417 & 38 & 18 & 0,6481 & 0,0932 & 0,588 & 0,5467 & 14 & 10 & 44 & 32 \\
\hline 1 & 0,7368 & 0,84732 & 37,62 & 38 & 0,8207 & 0,035 & 0,603 & 0,5956 & 25 & 16 & 28 & 31 \\
\hline 1 & 0,6684 & 0,67891 & 30,02 & 20 & 0,8177 & 0,0447 & 0,63 & 0,6147 & 22 & 13 & 33 & 32 \\
\hline 2 & 0,4473 & 0,5526 & 24,7 & 12 & 0,8852 & 0,0441 & 0,566 & 0,5545 & 25 & 12 & 31 & 32 \\
\hline 2 & 0,5631 & 0,79996 & 38 & 12 & 0,7246 & 0,0937 & 0,565 & 0,5458 & 21 & 10 & 37 & 32 \\
\hline 2 & 0,6315 & 0,79996 & 38,38 & 20 & 0,6011 & 0,1358 & 0,554 & 0,5325 & 17 & 9 & 44 & 30 \\
\hline 2 & 0,5421 & 0,54208 & 38,76 & 26 & 0,5661 & 0,1576 & 0,557 & 0,5285 & 21 & 10 & 36 & 33 \\
\hline 2 & 0,5263 & 0,5526 & 37,62 & 14 & 0,6433 & 0,1132 & 0,584 & 0,5686 & 24 & 10 & 28 & 38 \\
\hline 2 & 0,4 & 0,60523 & 36,1 & 23 & 0,7417 & 0,1152 & 0,565 & 0,5417 & 28 & 9 & 26 & 37 \\
\hline 2 & 0,5316 & 0,62102 & 38 & 29 & 0,7808 & 0,1158 & 0,541 & 0,508 & 27 & 12 & 20 & 41 \\
\hline
\end{tabular}


Anexo, cont.

Enclosure, cont..

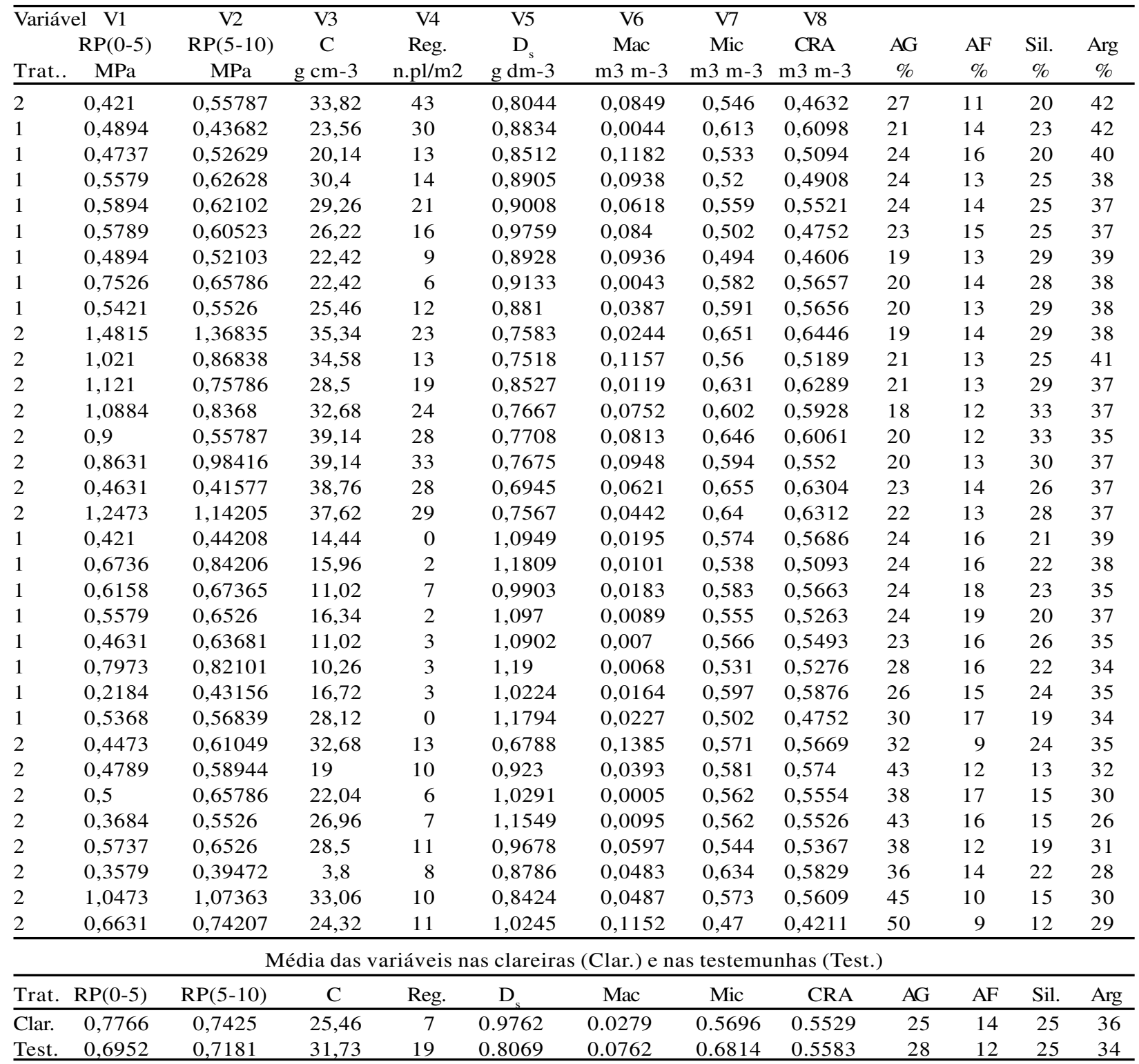

Em que:

Trat. 1 = testemunha; Trat. 2 = clareira

$\mathrm{RP}(0-5)=$ resistência à penetração entre 0 e $5 \mathrm{~cm}(\mathrm{MPa})$;

$\mathrm{RP}(5-10)=$ resistência à penetração entre 5 e $10 \mathrm{~cm}(\mathrm{MPa})$;

$\mathrm{C}=$ conteúdo de carbono no solo $\left(\mathrm{g} \mathrm{cm}^{-3}\right)$;

Reg.= quantidade de regeneração natural em cada amostra $\left(n^{\circ} \cdot\right.$ plantas $\left./ \mathrm{m}^{2}\right)$;

$\mathrm{D}_{\mathrm{s}}=$ densidade do solo $\left(\mathrm{g} \mathrm{dm}^{-3}\right) ; \operatorname{Mac}=\operatorname{macroporosidade}\left(\mathrm{m}^{3} \mathrm{~m}^{-3}\right) ;$ Mic $=\operatorname{microporosidade}\left(\mathrm{m}^{3} \mathrm{~m}^{-3}\right) ;$

$\mathrm{CRA}=$ capacidade de retenção de água $\left(\mathrm{m}^{3} \mathrm{~m}^{-3}\right)$;

$\mathrm{AG}=$ areia grossa $(\%)$;

$\mathrm{AF}=$ areia fina $(\%)$

Sil. = silte $(\%)$; e,

Arg. = argila $(\%)$ 
